\begin{tabular}{c} 
International Journal of Scientific World, $5(2)(2017) 96-105$ \\
International Journal of Scientific World \\
SPC \\
Website: $\begin{array}{c}\text { www. sciencepubco.com/index. } h \text { h } / I J S W \\
\text { doi: } 10.14419 / i j s w . v 5 i 2.7900 \\
\text { Research paper }\end{array}$ \\
\hline
\end{tabular}

\title{
Zika virus, the way forward: Nigeria as case study
}

\author{
MODO Emmanuel U ${ }^{1 *}$, OKORO Favour ${ }^{1}$, ORJI Blessing $\mathrm{O}^{2}$, NJOKU Rex-Clovis $\mathrm{C}^{1}$ \\ ${ }^{1}$ Department of Biochemistry, Madonna University, Nigeria. Elele campus. Rivers State, Nigeria \\ 2 Department of Biochemistry, University of Benin, Nigeria \\ *Corresponding author E-mail: emmanuelmodo@yahoo.com
}

\begin{abstract}
Zika Virus is a flavivirus that is responsible for an unprecedented current epidemic. It is an emerging mosquito borne virus that is a (+) sense single-stranded RNA virus from the Flaviviridae virus family, Flavivirus genre and the Zika virus specie. Zika virus bears a complex genome and virion structure. Its reproductive cycle in host cell involves virion endocytosis that allows entrance into host cell. Zika virus infection gives symptoms that are usually mild and last for few days and infection can be easily transferred from infected mosquitoes, from a pregnant woman to her fetus, sexual intercourse, blood transfusion, and laboratory exposure. Several methods are made available for the diagnosis of this infection and several ways to prevent this infection such as elimination and control of mosquito, prevention of mosquito bites and public awareness about Zika and mosquitoes. No vaccines are available for treatment yet but common medications are advised for symptoms. The virus has been associated with fetal microcephaly in humans. Information is provided for the epidemiology of Zika virus to evaluate the level of risk for people who may be planning to travel to or are recently returning from areas with possible local transmission. In the African region this is done considering a number of factors and cases in the past. The rapid development of a safe and effective vaccine, genetically modified mosquitoes, use of bacteria, and other innovations are currently worked on to curb the spread of Zika virus.
\end{abstract}

Keywords: Bacteria; Genome structure; Microcephaly; Nigeria; Zika Virus.

\section{Brief history}

Zika virus is an emerging mosquito borne virus that was first identified in Uganda in 1947 in rhesus monkeys through a monitoring network of sylvatic yellow fever. It was subsequently identified in humans in 1952 in Uganda and the United Republic of Tanzania. It was later identified in humans in 1968 for the first time in Nigeria. Outbreaks of Zika virus disease have been recorded in Africa, the America, Asia, and the Pacific [1].

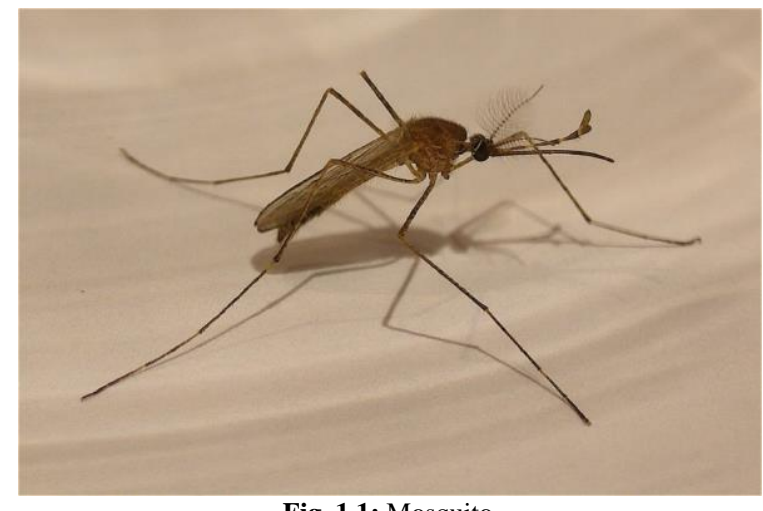

Fig. 1.1: Mosquito.

\section{Classification and taxonomy of zika virus}

i) GroupIV: (+) sense single-stranded RNA virus

ii) Virus family: Flaviviridae iii) Genre: Flavivirus

iv) Species: Zika virus

Vector: Aedes mosquitoes (which usually bite during the morning and late afternoon/evening hour) [2].

\section{Genome structure of zika virus}

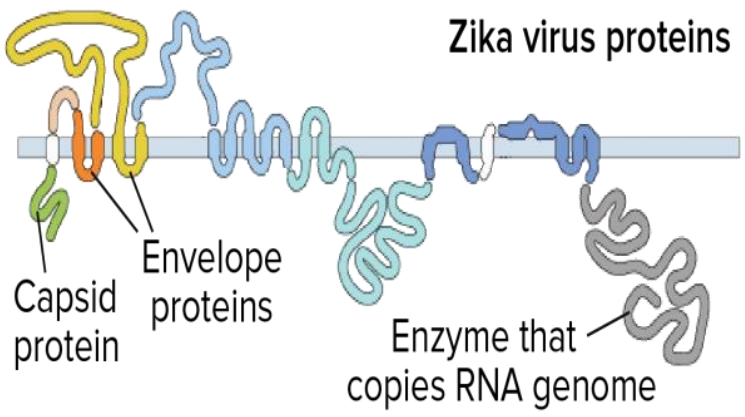

Fig. 1.2: Zika Virus Genome.

The Zika virus is a positive sense single-stranded RNA with two non-coding flanking regions known as the 5' NCR and the $3^{\prime}$ NCR (Non-Coding Regions). The open reading frame of the Zika virus reads as follows: 5'-C-prM-E-NS1-NS2A-NS2B-NS3-NS4ANS4B-NS5-3' and codes for a polyprotein that is subsequently cleaved into capsid (C), precursor membrane (prM), envelope (E), and non-structural proteins (NS). The E protein composes the majority of the virion surface and is involved with aspects of replication such as host cell binding and membrane fusion. NS1, NS3, and NS5 are large, highly-conserved proteins while the NS2A, 
NS2B, NS4A, and NS4B proteins are smaller, hydrophobic proteins. Located in the 3' NCR are 428 nucleotides that may play a part in translation, RNA packaging, cyclization, genome stabilization, and recognition. The 3 ' NCR forms a loop structure and the 5 NCR allows translation via a methylated nucleotide cap or a genome-linked protein [2].

\section{Virion structure of zika virus}

The structure of Zika Virus follows that of other flaviviruses. It contains a nucleocapsid approximately $25-30 \mathrm{~nm}$ in diameter surrounded by a host membrane derived lipid bilayer that contains envelope proteins $\mathrm{E}$ and $\mathrm{M}$. The virion is approximately $40 \mathrm{~nm}$ in diameter with surface projections that measure roughly $5-10 \mathrm{~nm}$
[4]. The surface proteins are arranged in an icosahedra-like symmetry [5].

\section{Mechanisim of action of zika virus (repro- ductive cycle of zika virus in a host cell)}

Once Zika virus particles are in the human body, they must enter individual cells in order to replicate and make more viruses. Cell entry is possible because a Zika virus particle carries specific proteins on its outer envelope that interact with receptor proteins on human cells. When the viral proteins bind to cell receptors, they "trick" the cells into taking up the viral particle.

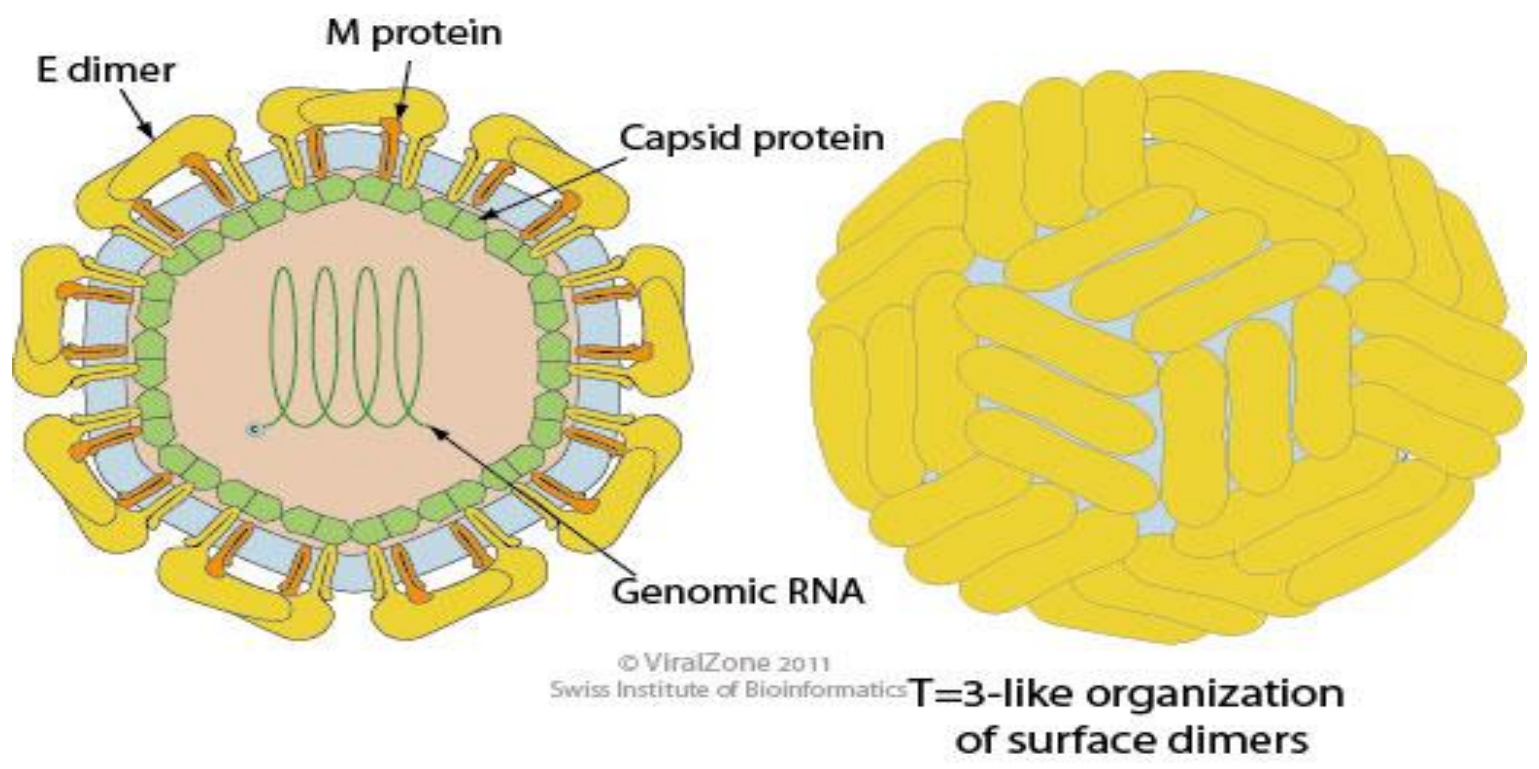

Fig. 1.3: General Structure of a Flavivirus Virion [3]

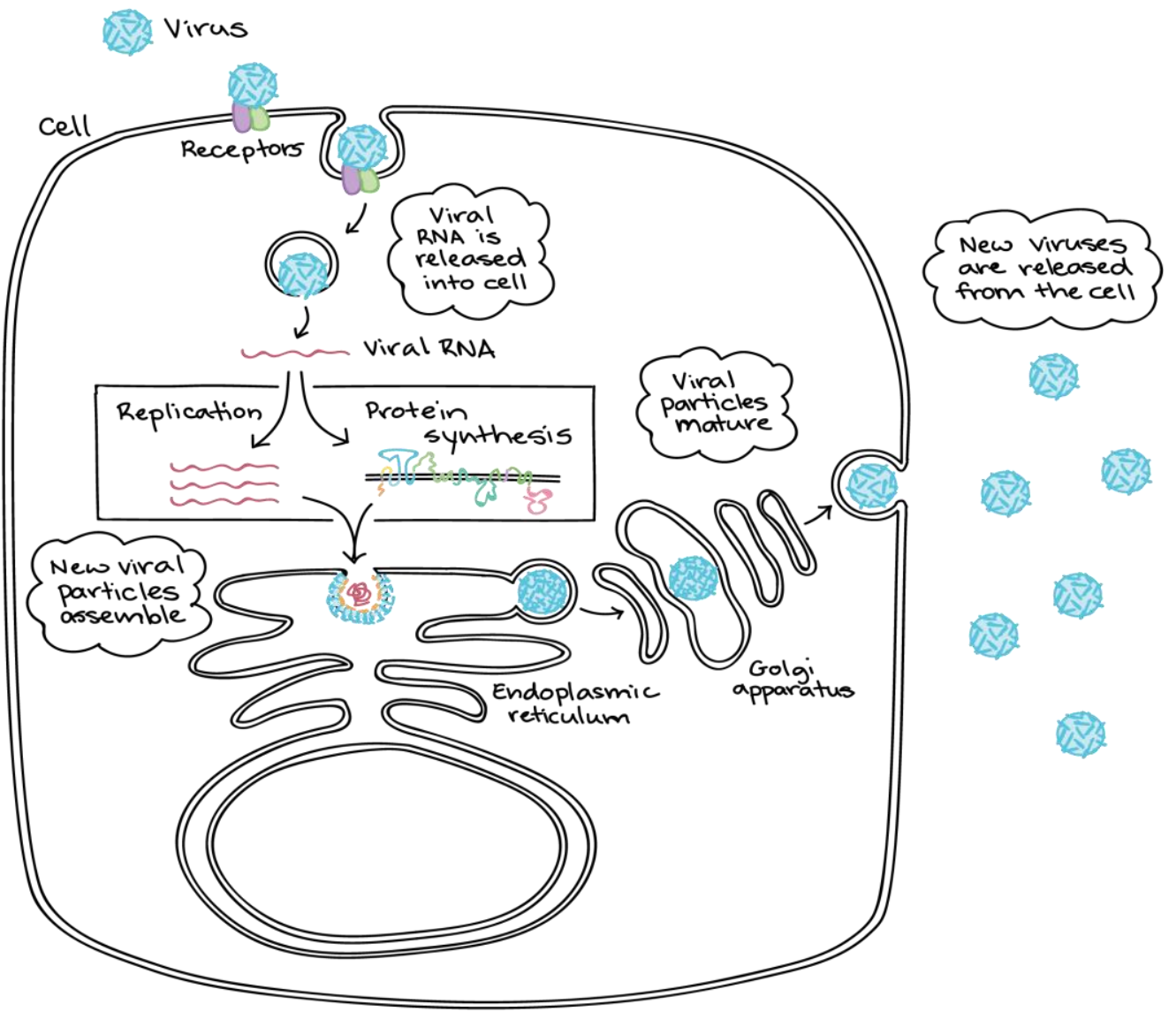

Fig. 1.4: The Flavivirus Life Cycle [6]. 
Inside the cell, the RNA genome of the virus is released into the cytoplasm, or fluid-filled main compartment, of the cell. There, the RNA molecule is "read" (translated) by enzymes in the cell to make a long protein, which are chopped up into a number of smaller proteins. Some of these proteins are the structural components needed to make new viral particles, such as capsid and envelope proteins. Other viral proteins copy and process the RNA genome.

Viral proteins and copies of the RNA genome assemble at the surface of the endoplasmic reticulum, a membrane compartment that is part of the cell's export system. New viral particles bud off into the interior of the ER, taking a small patch of ER membrane along with them. This "stolen" membrane will form the viral envelope. The particles then travel through another structure, the Golgi apparatus, where they undergo more processing before release at the cell surface. Released viral particles can infect other cells, continuing the infection cycle.

\section{Epidemiology}

Information on the level of Zika transmission is useful for public health professionals to evaluate the level of risk for people who may be planning to travel to or are recently returning from areas with possible local transmission.

A revised scheme has been developed by WHO, in collaboration with the US CDC and ECDC, to categorize the epidemiological profile of vector-borne Zika virus transmission in countries and territories. The classification can be applied at the national level as well as sub-national level when the necessary information is available. ECDC has adapted the classification scheme to reflect the risk to travelers more accurately [7].

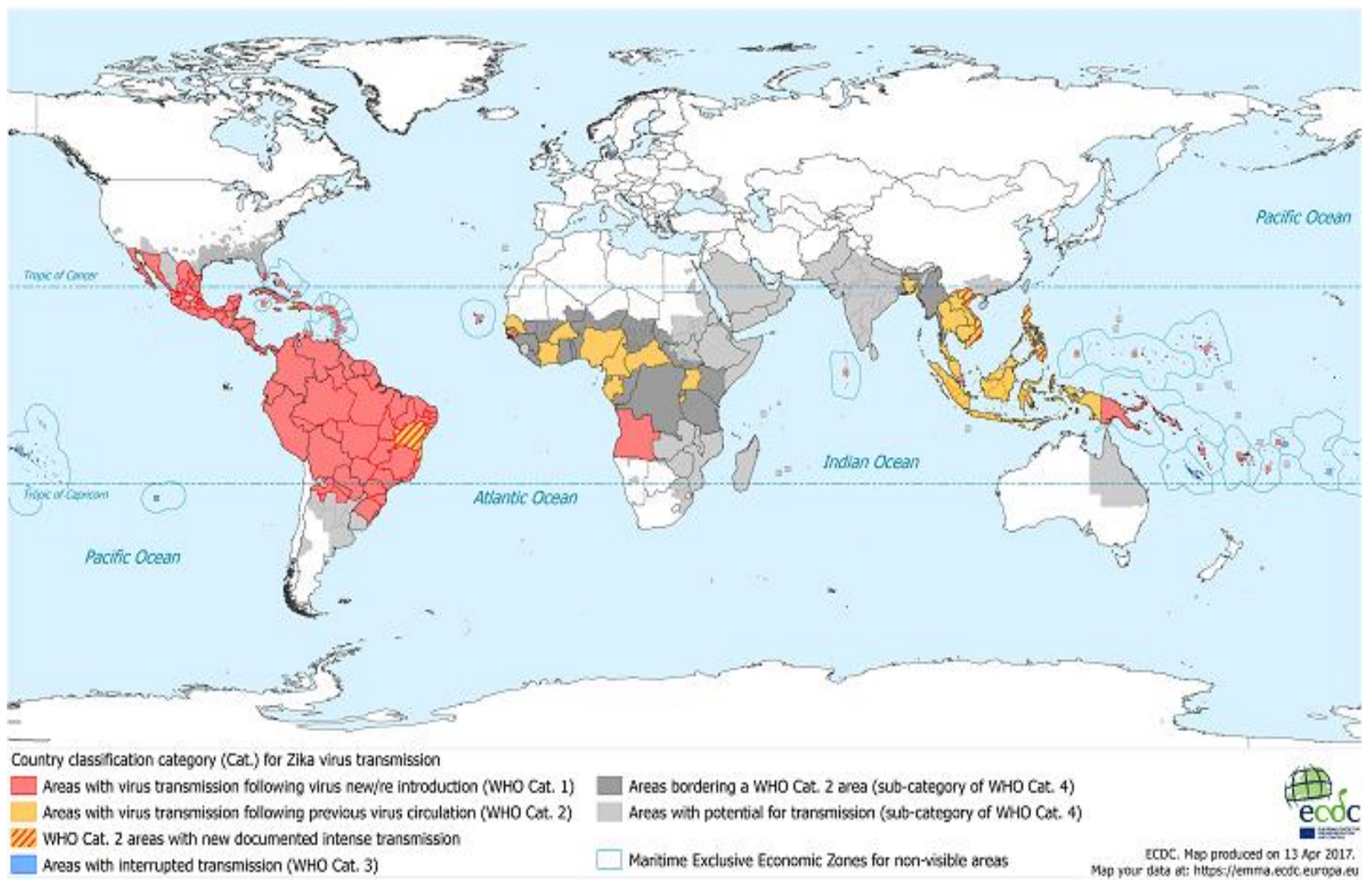

Fig. 2.1: ECDC Map [7].

To access the risk of a Zika outbreak in the countries of the WHO African region, consideration must be given to a number of ecological, epidemiological, structural and system factors that contribute to the likelihood and magnitude of an outbreak.

The main transmitting vector for Zika virus is Aedes aegypti mosquito. Historically, the sylvatic form of the virus was the main one reported in the few studies in Africa. As urbanization increased, however, the Aedes aegypti mosquito has adapted to and flour- ished in the urban environment breeding in open water containers and other collections of stagnant water. In many African cities, there is also a high proportion of the population who reside in slum areas where shelter, water storage, drainage and overall sanitation is poor, potentially increasing the availablility of breeding sites for the urban mosquitoes. 


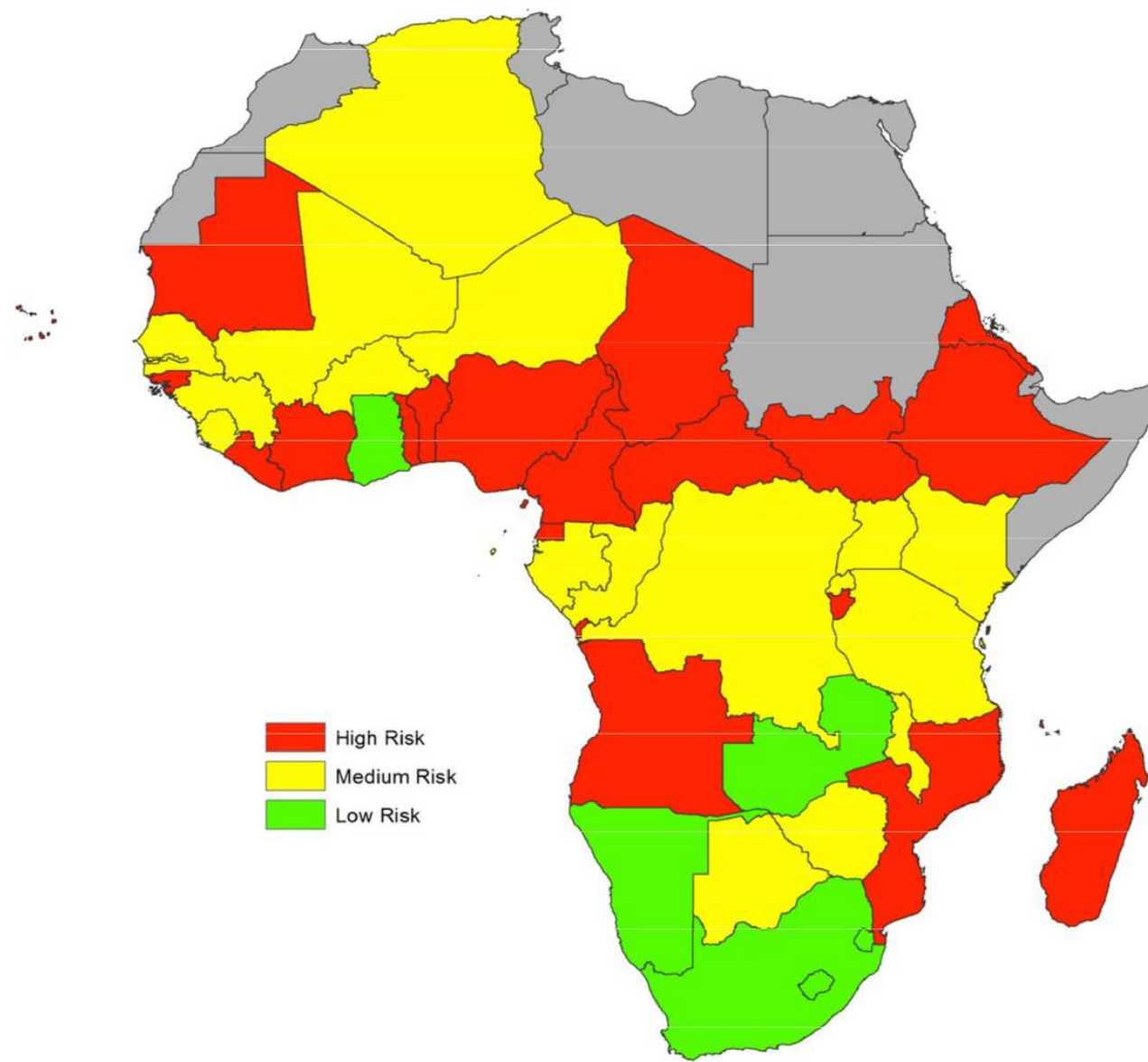

Fig. 2.2: Countries Ranked in Order of Increasing Risk of Zika Virus Epidemic Based on A Composite Index of Risk Derived from the Hazards, Vulnerabilities and Lacking of Coping Capacities [1].

Over time, African countries have become more connected with each other through land, water, and air transportation increasing the risk of disease spread. They have also increasingly become connected with other countries outside Africa, mainly through air transportation but also through shipping. The potential risk of importation of infections such as Zika virus from other countries is therefore high [1].

The current epidemiology of Zika in Nigeria has not been well documented or understood due to paucity of recent data. The virus shares a similar vector; the Aedes (Steogmyia) mosquitoes, also responsible for other flavivirus infections recorded in Nigeria such as yellow fever and dengue. Also, the environmental and human behavioral risk factors in areas with reported Zika outbreaks are similar to those found in Nigeria and would thus favour the circulation of Zika [8].

\section{Cases of Zika virus in Nigeria}

In 1954, a case of a 10 year old African female with fever and headache, this occurred during an epidemic of jaundice in Nigeria [9].

In 1956, it was experimentally induced in a 34year old European male (human volunteer) residing in Nigeria for $4 \frac{1}{2}$ months before inoculation. Symptoms included headache and fever [10].

In 1968, Zika virus was isolated from 3 febrile children aged 10 months, $2 \frac{1}{2}$ years and 3 years respectively [11].

In 1979, a case of a $2^{1 / 2}$ year old boy with fever and a 10 year old boy with fever, headache, and body pains. $40 \%$ persons tested has neutralizing antibodies to Zika virus (more frequently in younger people), demonstrating high prevalence of immunity in Nigeria [12]

As recent as 2014, the most recent outbreak of Ebola was recorded [13].

\section{Management of zika virus}

\subsection{Pathogenesis of zika virus}

Zika virus replicates in the mosquito's midgut epithelial cells and then its salivary gland cells. After 5-10 days, the virus can be found in the mosquito's saliva. If the mosquito's saliva is inoculated into human skin, the virus can infect epidermal karatinocytes, skin fibroblasts in the skin and the Langerhans cells. The pathogenesis of the virus is hypothesized to continue with a spread to lymph nodes and the bloodstream [14]. Flaviviruses generally replicated in the cytoplasm, but Zika antigens have been found in infected cell nuclei [15].

\subsection{Signs and symptoms}

The symptoms are similar to other arbovirus infections such as dengue, and include

i) Fever

ii) Skin rashes

iii) Conjunctivitis

iv) Muscle and joint pain

v) Malaise

vi) Headache [1].

These symptoms are usually mild and last for 2-7 days [16].

\subsection{Mode of transmission}

i) Zika virus is transmitted to people through the bite of an infected mosquito from the Aedes genus, mainly Aedes aegypti in tropical regions: This is the same mosquito that transmits dengue, chikungunya and yellow fever. These mosquitoes typically lay eggs in and near standing water in 
things like buckets, bowls, animal dishes, flower pots and vases. They prefer to bite people, and live indoors and outdoors near people. Mosquitoes that spread Zika are aggressive daytime biters, but they can also bite at night. Mosquitoes become infected when they feed on a person already infected with the virus. Infected mosquitoes can then spread the virus to other people through bites.

ii) Zika can be passed from a pregnant woman to her fetus: Infection during pregnancy can cause certain birth defects. A pregnant woman can pass Zika virus to her fetus during pregnancy. Zika is a cause of microcephaly and other severe fetal brain defects.

iii) Through sexual intercourse: Zika can be passed through sex, even if the infected person does not have symptoms at the time. The virus may also be passed by a person who carries the virus but never develops symptoms.

iv) Zika can be transferred through blood transfusion: There have been multiple reports of blood transfusion transmission cases in Brazil.

v) Through laboratory exposure: Prior to the current outbreak, there were four reports of laboratory acquired Zika virus infections, although the route of transmission was not clearly established in all cases [17].

\subsection{Diagnosis}

i) Polymerase Chain Reaction: Nucleic acid detection by reverse transcriptase-polymerase chain reaction (RT-PCR) targeting the non-structural protein 5 genomic region is the primary means of diagnosis. It is useful in the first 3-5 days after the onset of symptoms.

ii) Serological tests: An ELISA has been developed to detect IgM to Zika virus only after five days because it is closely related to dengue and yellow fever. It may cross-react with antibody tests for those viruses.

iii) Nucleic Acid Amplification Test: Nucleic acid amplification test (NAT) for detection of viral RNA can also be performed.

iv) Plaque Reduction Neutralization Assay: This has really improved specificity over immunoassays but may still yield cross-reactive results in secondary flavivirus infections [18].

\subsection{Prevention}

i) Elimination and control of mosquito: Avoid allowing standing water in outdoor containers so that they do not become mosquito breeding sites, avoid accumulating garbage, use mosquito nets in windows and doors.

ii) Prevention of mosquito bites: Personal protection measures to avoid mosquito bites should be applied when staying in risk areas, sleep under mosquito nets, and do not travel in affected areas.

iii) Public awareness about Zika and mosquitoes: People should be made aware about the disease and its preventive measures. They should take the basic precautions to protect themselves from the disease [18]

\subsection{Microcephaly, a case of zika virus}

Microcephaly is a neurological condition where an infant's head circumference is significantly smaller than the average size for infants of the same age. Microcephaly can lead to developmental delays in movement and speech among other [19].

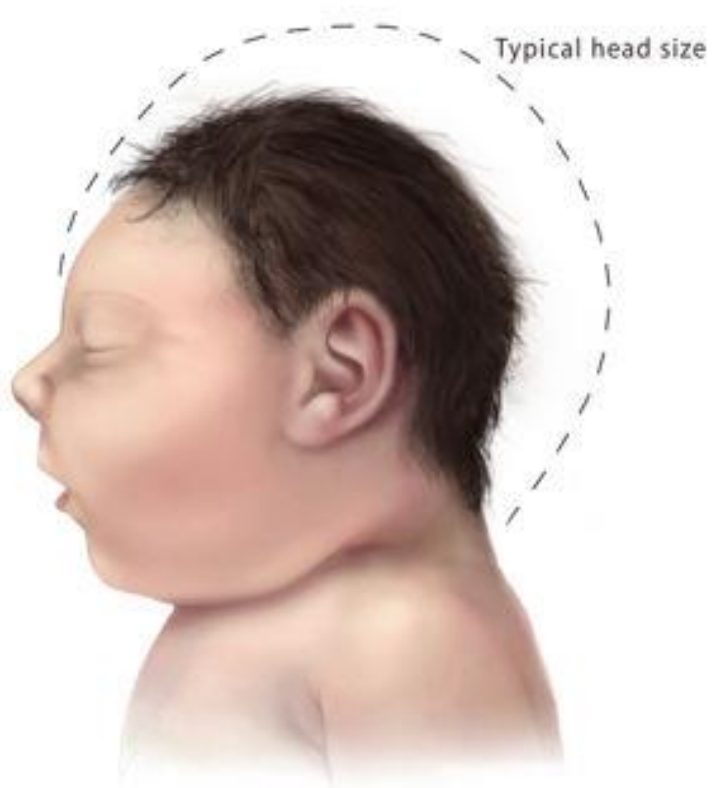

Baby with Microcephaly

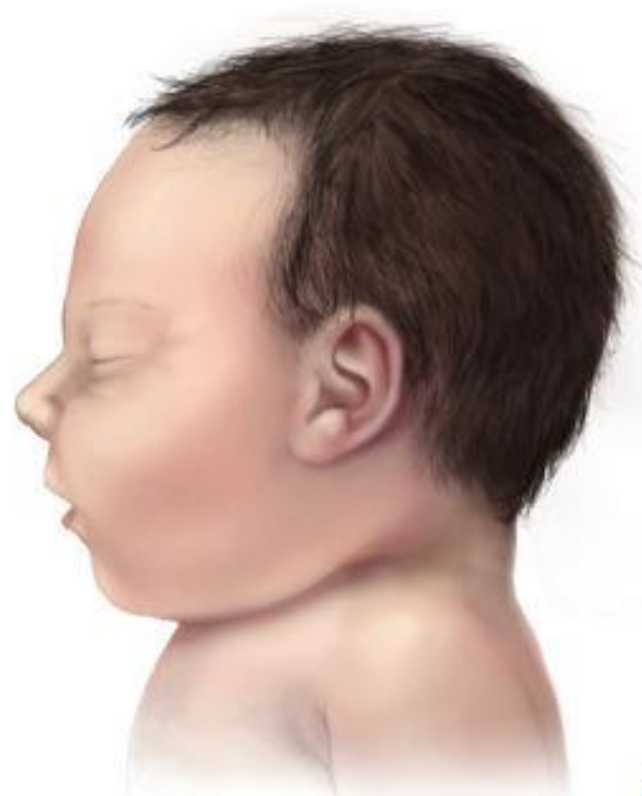

Baby with Typical Head Size

Fig. 3.1: Microcephaly-Comparison [20].

\subsubsection{Complications of microcephaly}

i) dwarfism or short stature

ii) facial distortion

iii) mental retardation

iv) hyperactivity

v) seizures 


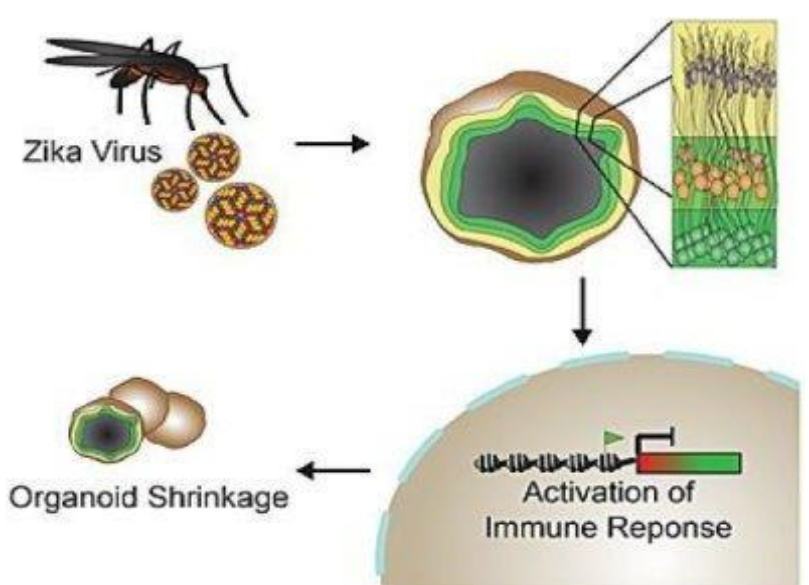

Fig. 3.2: Diagram Explaining Microcephaly [21].

In a 3-D brain model, Zika virus activates immune receptor TRL3, which in turn inhibits brain cell development and survival, causing the organoids to shrink (an effect reminiscent of microcephaly).

Using a 3D stem cell-based model of a first-trimester human brain, the team discovered that Zika activates TLR3; a molecule human cells normally use to defend against invading viruses. In turn, hyper-activated TLR3 turns off genes that stem cells need to specialize into brain cells and turns on genes that trigger cell suicide. When the researchers inhibited TLR3, brain cell damage was reduced in this organoid model.

In the study, Rana's team first made sure their organoid model was truly representative of the early developing human brain. They found that the model's stem cells differentiate into the various cells of the brain in the same way that they do in the first trimester of human development. The researchers also compared patterns of gene activation in organoid cells to a database of human brain genetic information. They found that, genetically speaking, their organoid model closely resembled fetal brain tissue at eight to nine weeks post-conception.

When the team added a prototype Zika virus strain to the 3D brain model, the organoid shrank. Five days after the infection, healthy, mock-infected brain organoids had grown an average of $22.6 \%$. In contrast, the Zika-infected organoids had decreased in size by an average of $16 \%$.

Rana's team also noticed that the TLR3 gene was activated in the Zika virus-infected organoids. TLR3 is a protein found both inside and attached to the outside of cells. TLR3's only job is to act as an antenna, sensing double-stranded RNA specific to viruses. When viral RNA binds TLR3, it kicks off an immune response. To do that, TLR3 helps activate many different genes that aid in fighting an infection. However, in developing brain cells, the researchers found TLR 3 activation also influences 41 genes that add up to a double whammy in this model (diminished stem cells differentiation into brain cells and increased cell suicide, a carefully controlled process known as apoptosis).

To determine whether TLR3 activation could be the cause of Zikainduceorganoid shrinkage, and therefore perhaps microcephaly, or merely a symptom of it, Rana's team treated some of the infected organoids with a TLR3 inhibitor, significantly tempered Zika virus' severe effects on brain cell health and organoid size, underscoring TLR3's role linking infection and brain damage.

However, the treated organoids were not perfect. As evidenced by their non-smooth outer surfaces, infected but treated organoids still encountered more cell death and disruption than uninfected organoids.

While promising, this research has been conducted only in human and mouse cells growing in the laboratory thus far. In addition, the Zika virus strain used in this study originated in Uganda, while the current Zika outbreak in Latin America involves a slightly different strain that originated in Asia [21].

\section{Treatment}

\subsection{Management therapy}

Presently there is no vaccine for Zika virus or treatment for Zika fever. Because symptoms are relatively mild and the disease is self-limiting, only supportive therapy is employed.

i) People sick with Zika virus should get plenty of rest and remain hydrated.

ii) Treat pain and fever with common medicines.

iii) If symptoms worsen, they should seek medical care and advice [1].

\subsection{Zika vaccines}

Two types of experimental Zika vaccines, a DNA vaccine and an inactivated virus vaccine, were each able to completely protect mice with one dose:

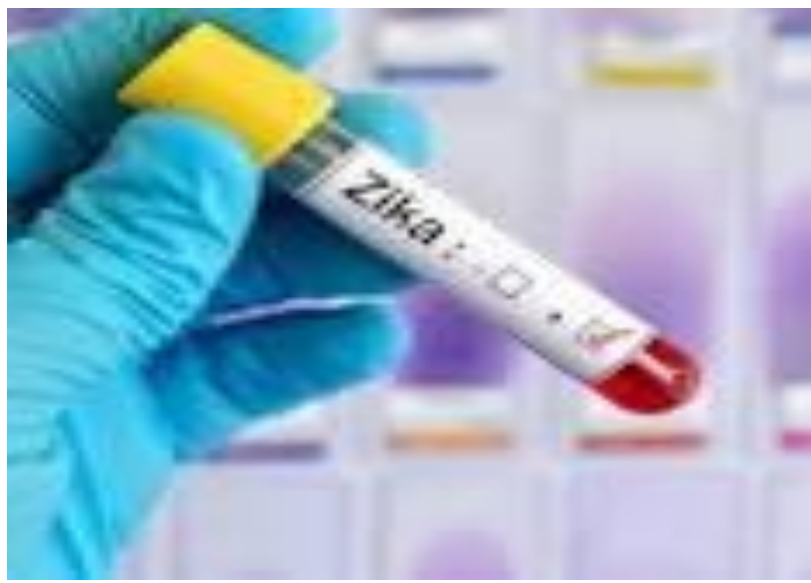

Fig. 3.3: Zika Vaccine.

\subsubsection{DNA vaccine}

The FDA just approved the world's first clinical trial of a Zika vaccine. The test will determine whether or not the vaccine is safe for use in normal, healthy people. However, they will not be able to determine whether or not the vaccine prevents the disease.

The vaccine, called GLS-5700, has been shown to cause a strong antibody response against the Zika virus in monkeys and mice. The trial will be run by Inovio Pharmaceuticals, a company specializing in immunotherapy and GeneOne Life Science, a DNA vaccine developer. It will begin in just a few short weeks, and it will include 40 adult subjects. If successful, the vaccine may be tested in people who have an existing Zika infection in later trials. GLS-5700 is what is known as a "DNA vaccine", a relatively new approach to fighting disease. Instead of directly injecting parts of the infectious agent, it consists of DNA coded to produce a special protein the same way as a normal shot, and it is also zapped with a device that delivers a short electrical pulse to help guide the DNA into the patient's cells. Once this process is complete, the new DNA trains the immune system of the patient to fight the disease [22].

This may be the first Zika vaccine approved for testing, but it is not the only one being developed. The National Institute of Allergy and Infectious Diseases is also in the process of developing a DNA vaccine, which may begin phase 1 testing as soon as August. However, it is important to note that clinical testing is a long and complex process, and that it might still be years yet before either of these vaccines is ready for the mass market.

Generally the DNA snippets included in such vaccines are viral blueprints for the unique proteins that cloak a particular virus particle. Smuggled into human cells, the code can be deciphered and used to train the immune system to spot the virus by its distinctive outerwear [23]. 


\subsubsection{Inactivated vaccine}

Researchers use whole microbes, killed by heat, radiation, or chemicals (in this case by a chemical treatment) and offer them to the immune system for training.

In the mice, both vaccines spurred the rodents' immune systems to create 'neutralizing antibodies' - proteins that could accurately identify Zika virus particles and raise immune responses strong enough to take out the virus [23].

\subsection{New IBM-designed macromolecule}

The new IBM-designed macromolecule could be a 'magic bullet' in the fight against viruses. Viruses are difficult to treat because they mutate very quickly by the time the drug is developed, it may no longer work. That is why IBM Research teamed up with the Institute of Bioengineering and Nanotechnology (IBN) to design a macromolecule that can treat viruses no matter how much they mutate. The researchers tested their macromolecule against Zika, Ebola and Dengue fever with success.

Dr Yi Yan Yang of IBN said "Viral diseases continue to be one of the leading causes of morbidity and mortality. We have created an anti-viral macromolecule that can tackle wily viruses by blocking the virus from infecting the cells, regardless of mutations. It is not toxic to collaboration with a global community of researchers."

The macromolecule works in three ways, or with a "powerful triple-play action".

i) First, it attracts the proteins on viruses so they will not infect cells that are healthy.

ii) Secondly, it prevents the virus from growing by bolstering the body's immune system.

iii) Thirdly, it works to halt virus replication [24].

The researchers said the macromolecule could be incorporated in anti-viral wipes or detergent to help people avoid infections soon. In the future, the macromolecule could be utilized to develop a new way to vaccinate. The researchers focused on glycoprotein, which sits on the outside of all viruses and attach to cells in the body, allowing the viruses to do their dirty work by infecting cells and making us sick. Using that knowledge, the researchers created a macromolecule, which is basically one giant molecule made of smaller subunits. This macromolecule has key factors that are crucial in fighting viruses.

First, it is able to attract viruses towards itself using electrostatic charges. Once the virus is close, the macromolecule attaches to the virus and makes the virus unable to attach to healthy cells. Then it neutralizes the virus' acidity levels, which makes it less able to replicate.

As an alternative way to fight, the macromolecule also contains a sugar called mannose. This sugar attaches to healthy immune cells and forces them closer to the virus so that the viral infection can be eradicated more easily. The researchers tested out this treatment in the lab on a few viruses, including Ebola and dengue, and they found that the molecule did work as they thought it would. According to the paper, the molecules bound to the glycoproteins on the viruses' surface and reduced the number of viruses. Further, the mannose successfully prevented the virus from infecting immune cells [25].

\subsection{Harvard's cheap paper zika test}

Harvard's cheap paper Zika test can detect the virus in hours instead of weeks. They developed a paper device with biology-based sensors that can quickly diagnose the Zika virus. The development could be incredibly valuable in parts of the world where Zika is spreading quickly and healthcare workers have to wait weeks to confirm whether someone has contracted the dangerous condition.

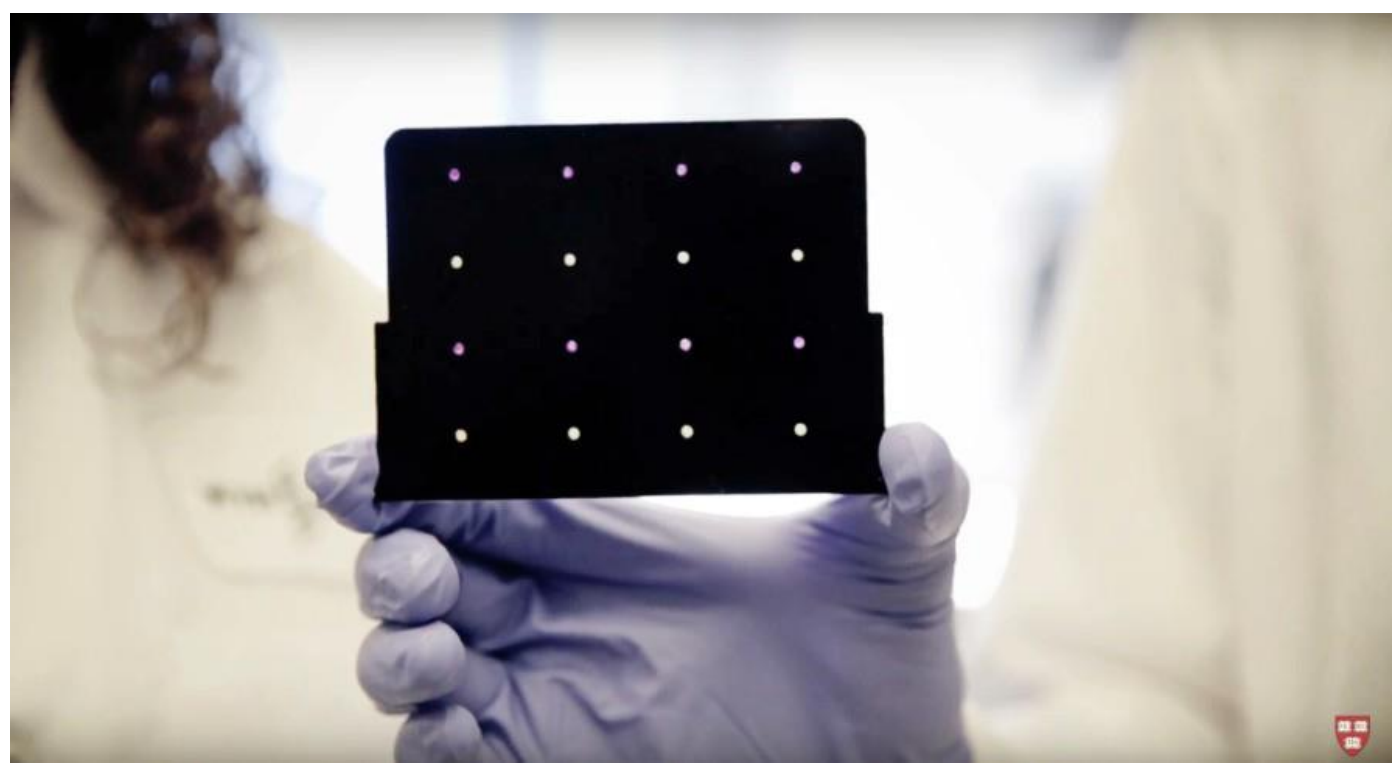

Fig. 3.4: Cartridge with Paper Strips

The Wyss Institute at Harvard University developed a cartridge with paper strips coded with a synthetic network of genes that react to the presence of certain microbes. A color change, visible to the naked eye, takes place if the RNA sequence associated with $\mathrm{Zika}$ is detected. The whole process takes place within two to three hours. As it stand right now, healthcare workers must rely on blood samples to test for Zika, and results can take days or weeks to return. Current tests also cannot distinguish between zika and dengue, but Harvard's technology can. The tool can also detect the virus in samples of urine and saliva, as well as blood [26].

\section{Recent findings to avoid further spread of the virus}

\subsection{Bacteria helping to curb the spread of zika virus}

Researchers infected mosquitoes with bacteria called Wolbachia and released them to mate with non-Wolbachia-infected mosquitoes in the wild, and then measured levels of the Zika virus in the mosquitoes. Over a few generations, the virus levels dropped and 
became inactive, which means those mosquitoes cannot transmit the Zika virus to humans.

The idea to use Wolbachia-infected mosquitoes to control Zika virus outbreaks is based in common sense. The mosquito vector Aedes Aegypti which carries the Zika virus is also responsible for spreading malaria, dengue fever and yellow fever. Previous trials using Wolbachia to reduce dengue fever seemed promising, spurring scientists in Brazil to try it out on in Zika hotspots, where thousands of children have been born with microcephaly, a birth defect resulting in an abnormally small head and brain.

Male mosquitoes are infected with the Wolbachia bacteria and then released to mate with non-Wolbachia females. That union results in infertile eggs in female offspring, so after a few generations, the number of disease-carrying mosquitoes falls dramatically. The bacteria are passed on from generation to generation, translating into a long-term strategy for reducing the number of disease-carrying mosquitoes in regions where the infected males are released. Because mosquitoes have a short life span, it only takes a few months to see the impact over several generations [27].

\subsection{Genetically altered mosquitoes are being released to fight zika}

Oxitec genetic control works by inserting a gene into the target organism, which prevents the insect from surviving to adulthood. The pest control gene produces a protein called tTAV (tetracycline repressible activator variant that has been optimises to only work in insect cells. In the engineered insects, when the tTAV gene is expressed, the non-toxic protein ties up the cell's machinery so it is other genes are not expressed and the insect dies. The proteins produced are non-toxic in the insects, so if any animals eat them it would be the same as eating a wild insect - they will be digested in just the same way that all other insects are digested.

But how do we produce insects if they die? There's an antidote given to the insects in the rearing facility that acts like a switch to turn off the tTAV gene preventing the tTAV protein from working. This antidote, tetracycline, an anitibiotic, binds to the tTAV protein and disables it. So in the presence of the antidote, the Oxitec insects are able to survive and reproduce in the rearing facility, but when the males are released into the wild, their offspring cannot access the anibiotic in the quantities needed to survive, so they die before reaching adulthood.

The self-limiting gene compromises tetO (tetracycline Operator) sites, which bind tTAV (tetracycline repressible Trans-Activating factor Variant) protein, a promoter, and coding sequence for tTAV Without the tetracycline antidote, tTAV protein is produced, which simultaneously binds to transciptional machinery and tetO sites, thereby enhancing the expression of the self-limiting gene. This positive feedback system produces large amounts of tTAV, which binds to more and more transcriptional machinery unavailable for other essential gene expression. The inhibition of essential gene expression leads to cell death and the death of the insect before it reaches adulthood. When tetracycline is added to the larval aquatic diet, it binds and inactivates tTAV, switching off the positive feedback system. The transcriptional machinery is not depleted as only a small amount of tTAV is produced, which does not affect normal cell function, and the insects survives to reproduce naturally in the production facility [28].

\subsection{Billboard imitates human sweat to snare mosqui-} toes

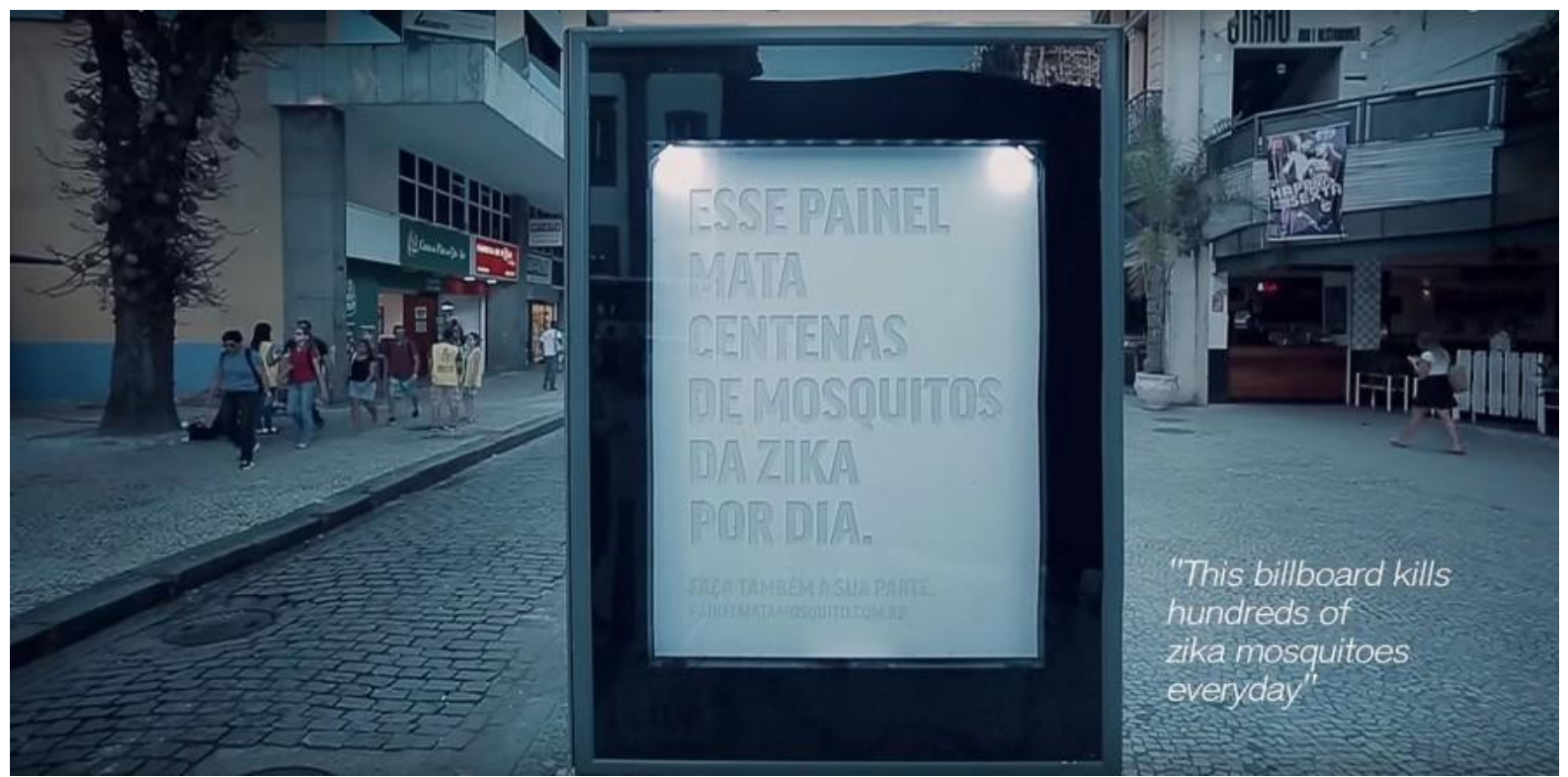

Fig. 4.1: Mosquito Billboard.

With the Zika virus threatening humanity, two advertising companies decided to get involved. Posterscope and NBS designed the mosquito killer billboard to bait and kill mosquitoes in Brazil. The billboards release carbon dioxide and lactic acid to imitate human breath and sweat, and they are equipped with fluorescent lights to increase their appeal to the troublesome bugs. The agencies say mosquitoes can smell the signs from more than a mile away. When they arrive at the panel ready to take a bite, they zoom into a catch mechanism which draws the insects inside the panel. Inside, they are unable to escape, dehydrate, and die. This board kills hundreds of zika mosquitoes every day [29].

10.4. Recycled tire traps are seven times more effective than traditional mosquito traps
The use of recycled materials is not only good for the environment Sometimes, it is simply the best way to get a job done. Such is the case with the ovillanta mosquito traps crafted from old tires and tested in Guatemala. Over the course of ten months, researchers supported by the Guatemala Ministry of Health's Vector Control Program observed the number of mosquito eggs collected by the simple traps. Based on their pending study, the ovillanta tire traps captured seven times the number of eggs as a standard trap.

The ovillanta traps are specifically deployed to capture mosquitoes of the Aedes genus. These mosquitoes are those notoriously known for transmitting devastating viruses, such as Zika, dengue, chikungunya, and yellow fever. Aedes mosquitoes can become resistant to pesticides and although adults only live for about two to four weeks, eggs can remain viable for up to a year. This ena- 
bles populations to mitigate the effects of hazardous weather. Many communities lack the resources to deal with this resilient pest and are particularly vulnerable to outbreaks of mosquitoborne diseases.

The ovillanta is composed of two 20-inch segments of a used car tire, which is joined together to create a basin. "We decided to use recycled tires - partly because tires already represent up to $29 \%$ of the breeding sites chosen by the Aedes Aegypti mosquitoes, partly because tires are a universally affordable instrument in lowresource settings, and partly because giving old tires a new use creates an opportunity to clean up the local environment." says lead researcher Gerardo Ulibarri.

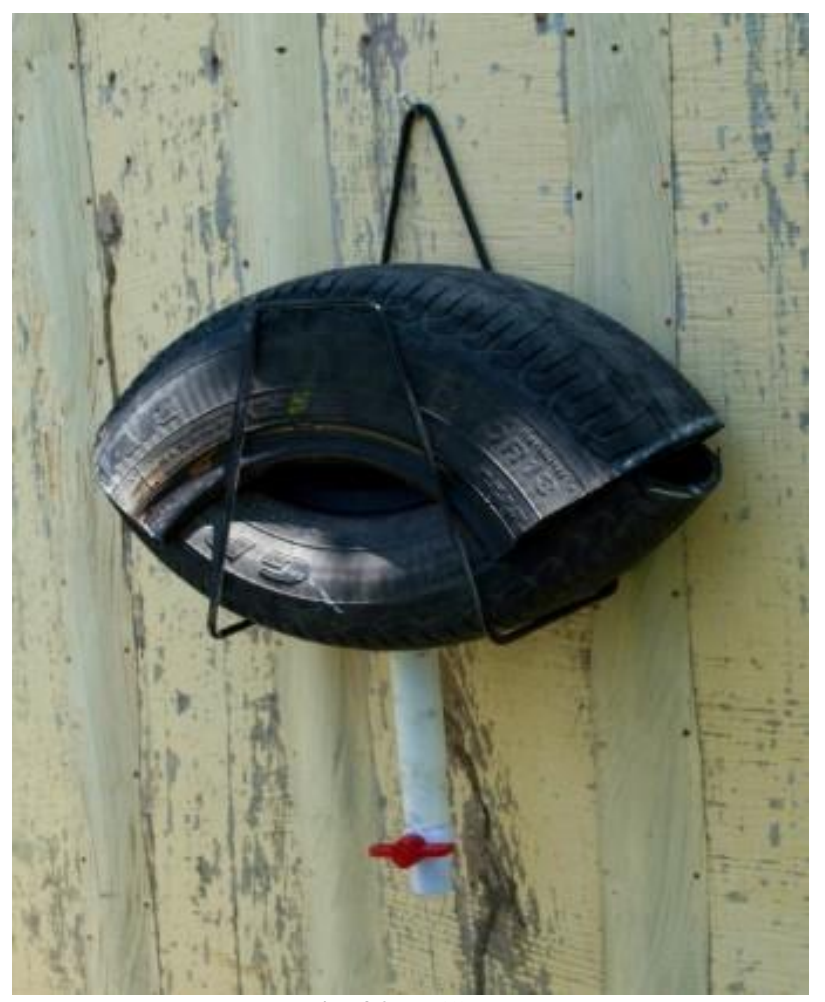

Fig. 4.2: Ovillanta.

The mosquitoes are lured into the tire's basin with non-toxic substance that includes a special mosquito pheromone, which signals to pregnant mosquitoes that this is a safe place to lay their eggs. The eggs are then dropped on a small "raft" floating in the solution, which is removed twice a week so that the eggs may be removed and destroyed. This low-tech solution costs only a fraction of traditional techniques and does not harm other animals in the process of catching the mosquitoes [30].

\section{Conclusion}

Zika virus is easily transmitted as a result of Aedes Aegypti mosquito, the main vector, being a household pest. The virus is known to cause mild symptoms when one is infected. Microcephaly is the only found major disease Zika virus causes which is prevalent in new borns. Researches are presently on for production of vaccines for Zika virus and a faster method for detection of the virus has also been produced. Several other measures have been taken for proper control of mosquitoes such as the tire traps, genetically altering of mosquitoes, billboards for trapping mosquitoes, and the use of bacteria for control of mosquito population.

\section{References}

[1] World Health Organization. A technical report on Zika Virus Risk Assessment in the WHO African Region (2016).

[2] O. Faye, C. Freire, A. Lamarino, O. Faye, J. de Oliveira, M. Diallo, A. Sall, Molecular evolution of zika virus during its emergence in the 20th century. PLOS Neglected Tropical Diseases (2014) https://doi.org/10.1371/journal.pntd.0002636.

[3] G.S. Campos, A.C. Bandeira, S.I. Sardi, Zika virus outbreak, Bahia, Brazil. CDC Emerging Infectious Diseases (2015). Retrieved from https://microbewiki.kenyon.edu/index.php/File:FlavivirusStructure Zika.jpeg on 27/03/2017

[4] T. Chambers, C. Hahn, R. Galler, C. Rice, Flavivirus genome organization, expression, and replication. Annual Reviews of Microbiology https://doi.org/10.1146/annurev.mi.44.100190.003245.

[5] Swiss Institute of Bioinformatics (n.d.). Flaviviridae. Retrieved from https://microbewiki.kenyon.edu/index.php/Zika_virus on $12 / 02 / 2017$.

[6] Khanacademy, The biology of zika virus (2016). Retrieved from https://www.khanacademy.org/science/biology/biology-ofviruses/virus-biology/a/what-is-zika-virus on 29/03/2017.

[7] European Centre for Disease Prevention and Control, Zika Epidemics 2014 onwards: Current Zika transmission (2017). Retrieved from

http://ecdc.europa.eu/en/healthtopics/zika_virus_infection/zikaoutbreak/Pages/zika-outbreak.aspx on 30/03/2017.

[8] Nigerian Center for Disease Control. Public Health risk assessment of zika virus in Nigeria and interim recommendations (2016). Retrieved from http://www.ncdc.gov.ng/diseases/guidelines on 01/04/2017.

[9] F.N. MacNamara, Zika virus: a report on three cases of human infection during an epidemic of jaundice in Nigeria. Trans R Soc Trop Med Hyg (1954) 48(2):139-45 https://doi.org/10.1016/00359203(54)90006-1.

[10] W.G.C. Bearcroft, Zika virus infection experimentally induced in a human volunteer. Trans R Soc Trop Med Hyg (1956) 50(5):442-48 https://doi.org/10.1016/0035-9203(56)90090-6.

[11] D.L. Moore, O.R. Causey, D.E. Carey, S. Reddy, A.R. Cooke, F.M Akinkugbe, et al. Arthropod-borne viral infections of man in Nigeria, 1964 -1970. Ann Trop Med Parasitol. (1975) 69(1):49-64. https://doi.org/10.1080/00034983.1975.11686983.

[12] A.H. Fagbami, Zika virus infections in Nigeria: virological and seroepidemiological investigations in Oyo State. J Hyg (Lond) (1979) 83(2):213-9 https://doi.org/10.1017/S0022172400025997.

[13] BBC News, Nigeria 'on red alert' over Ebola death in Lagos (2014). Retrieved from http://www.who.int/mediacentre/news/statements/2014/nigeriaends-ebola/en/ on 12/04/2017.

[14] D.M. Knipe, P.M. Howley, Fields Virology (5th ed.). Lippincott Williams \& Wilkins. (2007) 1156-1199

[15] A. Buckley, E.A. Gould, Detection of virus-specific antigen in the nuclei or nucleoli of cells infected with zika or langat virus. Journal of General Virologu, (1988) 69 (8): 1913-1920 https://doi.org/10.1099/0022-1317-69-8-1913.

[16] L.H. Chen, D.H. Hamer, Zika virus: Rapid spread in the western hemisphere. Annals of Internal Medicine (2016) 164 (9): 613 https://doi.org/10.7326/M16-0150.

[17] Centers for Disease Control and Prevention, Transmission and risks. Zika Virus (2016). Retrieved from www.cdc.gov/zika/transmission/index.html on 15/04/2017

[18] G. Dhurba, Zika virus: Structure, epidemology, pathogenesis, symptoms, laboratory, diagnosis, and prevention. Laboratoryinfo.com (2016). Retrieved from www.laboratoryinfo.com/zika-virusstructure-epidemiology-pathogenesis-symptoms-laboratorydiagnosis-and-prevention/ on 24/03/2017.

[19] Mayo Clinic Staff, Microcephaly (2016). Retrieved from www.mayoclinic.org/diseasesconditions/microcephaly/basics/definition/con-20034823 $10 / 04 / 2017$

[20] Centers for Disease Control and Prevention, Facts about microcephally (2016). Birth Defects. Retrieved from www.cdc.gov/ncbddd/birthdefects/microcephaly.html\#Microcephal lyCompareModal2 on 12/03/2017.

[21] University of California - San Diego, Zika virus may cause microcephally by hijacking human immune molecule (2016). Retrieved

www.sciencedaily.com/releases/2016/05/160506132202.htm on 26/03/2017.

[22] J.M. Rodriguez, Experimental zika vaccine to be tested on humans for the first time: Health innovation. Inhabitat (2016). Retrieved from www.inhabitat.com/experimental-zika-vaccine-to-be-testedon-humans-for-the-first-time/ on 23/03/2017.

[23] B. Mole, Experimental zika vaccines work in mice, protect with one shot: Scientific method / science \& exploration. Arstechnica (2016). Retrieved 
www.arstechnica.com/science/2016/06/experimental-Zika-

vaccines-work-in-mice-protect-with-one-shot/.

[24] L. Cooke, New IBM-designed macromolecule could be a 'magic bullet' in the fight against viruses: Health news (2016). Retrieved from www.inhabitat.com/exciting-new-ibm-designed-macro molecule-could-change-how-we-fight-viruses/

[25] C. Maldarelli, IBM creates a molecule that could destroy all viruses. Popular Science (2016). Retrieved from www.popsci.com/macromolecule-developed-by-ibm-could-fightmultiple-viruses-at-once on 17/04/2017.

[26] K. Medlock, Harvard's cheap paper zika test can detect the virus in hours instead of weeks: Health news. Inhabitat (2016). Retrieved from www.inhabitat.com/harvard-develops-paper-based-test-forzika-more-accurate-than-a-blood-test/ on 11/04/2017.

[27] C. Distasio, Can bacteria help curb the spread of zika virus: Health news. Inhabitat (2016). Retrieved from www.inhabitat.com/bacteria-infected-mosquitoes-prove-effectivein-reducing-Zika-virus-transmission-rates/ on 11/02/2017.

[28] Oxitec, How the self limiting gene works (2016). Retrieved from www.oxitec.com/our-solution/technology/the-science/ 29/03/2017.

[29] L. Cooke, Millions of genetically altered mosquitoes are being released in the Cayman Islands to fight Zika: Health nature / environment. Inhabitat. (2016). Retrieved from www.inhabitat.com/millions-of-genetically-altered-mosquitoes-arebeing-released-to-fight-zika/ on 25/04/2017.

[30] B. Greg, Carousel showcase design. Inhabitat (2016). Retrieved from www.inhabitat.com/recycled-tires-redesigned-as-highlyeffective-mosquito-traps/ on 19/03/2017. 